\section{Regulation of kinase activity}

SIR-In a recent News and Views article, Hardie $^{1}$ described the emerging evidence that protein kinases can be inhibited by interacting with substrate-like sequences. These sequences are present either within the same molecule, as in kinase $\mathrm{C}$, or in a different molecule, as in the regulatory subunits of cyclic AMP-dependent kinases. I would like to point out that an inhibitory role of amino-terminal sequences over the carboxy-terminal catalytic domain has also been shown for $\mathrm{p} 60^{\mathrm{v}-\mathrm{s} r \mathrm{c}}$, a member of the non-receptor family of tyrosine kinases $^{2}$. The mechanism whereby this negative regulation occurs has not yet been addressed, although studies performed on $\mathrm{p} 60^{\mathrm{c}-\mathrm{s} r \mathrm{c}}$, the cellular (c) homologue of the viral (v) protein, support the idea of substrate-like sequences being involved.

Amino-acid positions 90 and 92 of $\mathrm{p} 60^{\mathrm{cssc}}$ are tyrosine residues, one of which is surrounded by acidic amino acids as are many tyrosine-kinase target sequences (see figure). These sequences are located in the amino-terminal modulatory domain of the molecule within a stretch of about 50 amino acids that are conserved among all the members of the $s r c$ family. This region has been called the $\mathrm{SH} 3$ or $\mathrm{A} \mathrm{box}^{3}$ and it is not present in the receptor class of tyrosine kinases.

Activated p $60^{c-s r c}$ molecules, such as those bound to middle- $T$ antigen of polyomavirus, or those present in certain neuroblastoma cell lines, are phosphorylated on tyrosine at their amino termini $i^{4.5}$. Based on their neighbouring sequences, tyrosine residues 90 and 92 represent the most likely targets of phosphorylation within this amino-terminal region. An activation of the kinase. interpretation of these results is that in the off state the catalytic carboxy-terminal domain interacts with the amino-terminal substrate-like sequences. Phosphorylation of the amino-terminal site would only occur when the kinase is activated, presumably by an induced conformational change. Phosphorylation at these sites may allow maintainance of the on state.

A similar effect is observed with certain amino-acid substitutions of $\mathrm{p} 60^{\mathrm{c}-s \mathrm{sc}}$. These are Gly $63 \rightarrow$ Asp, Arg $95 \rightarrow$ Trp, and Thr $96 \rightarrow$ Ile, which are present in the viral protein $\mathrm{p} 60^{\mathrm{v} s \mathrm{~s} c}$, and are sufficient to increase the specific activity of the kinase ${ }^{6}$. The recombinant chimaeras between $\mathrm{v}$ $s r c$ and c-src used in these studies ${ }^{7}$ are shown schematically in the figure. Among these three mutations, the crucial one is likely to be Arg $95 \rightarrow \operatorname{Trp}$, because Gly $63 \rightarrow$ Asp when present alone in $\mathrm{p} 60^{\mathrm{c}-\mathrm{sic}}$ is silent, and Thr $96 \rightarrow$ Ile is not conserved in other strains of Rous sarcoma virus. Arg 95 is three residues away from the tyrosine-phosphorylation site and its presence could weaken the interaction with the kinase domain, thereby releasing the inhibition and allowing constitutive

A more rigorous proof that Arg 95 lies within a kinase regulatory domain has been obtained by Potts et al. ${ }^{7}$, who find that the Arg $95 \rightarrow$ Trp mutation alone is sufficient to activate p $60^{\mathrm{c}-\mathrm{s} r}$. In addition, this group and also L. Fox, K. Frost and J.S. Brugge (personal communication), have shown that mutations at either tyrosines 90 or 92 , or the deletion of amino acids 92-95, activate the transforming potential and the kinase activity of $\mathrm{p} 60^{\mathrm{c}-\mathrm{sir}}$. Thus, it is possible that an intramolecular

\section{W \\ * * 95 \\ poo $^{\text {c-sre }}$ (amino acid 81-108) GGVTTFVAL YDYESRTETDLSFKKGE}

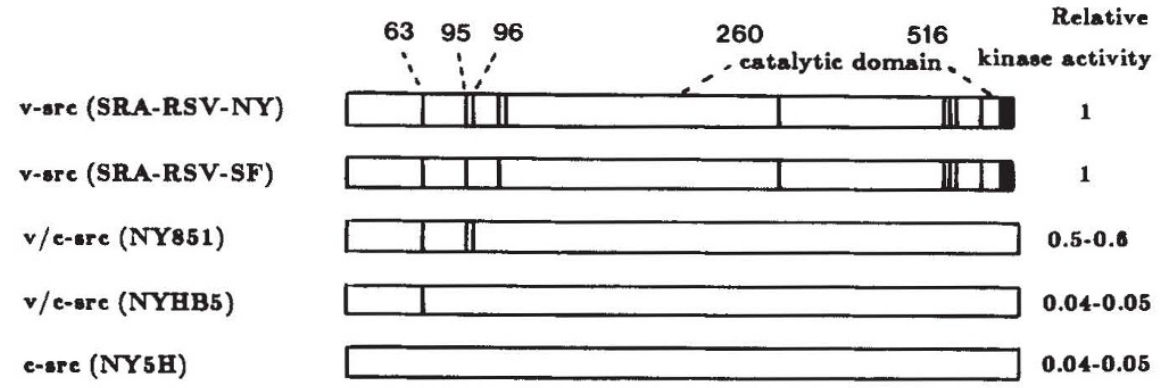

Amino acids 81 to 106 of $p 60^{c-s r c}$. W, substitution of $A r g \rightarrow \operatorname{Trp}$ as in $p 60^{v-s r c}$, asterisks are above tyrosines 90 and 92 , underlines, acidic amino acids surrounding the tyrosine-phosphorylation sites. Lower panel, schematic diagram of various src proteins from: Schmidt Ruppin subgroup A Rous sarcoma virus, New York strain (SRA-RSV-NY); San Francisco strain (SRA-RSV-SF); chimaeric viral and cellular src recombinant viruses (NY851 and NYHB5); a non-mutated celiular src recombinant virus (NY5H). Mutations 63, 95 and 96 are indicated; vertical lines show aminoacid substitutions in other regions of the viral protein as compared with the cellular src protein. The relative kinase activity was measured by in vitro phosphorylation of enolase as previously described ${ }^{5}$. negative control of kinase activity among the $s r c$ family resides within substratelike sequences outside the catalytic domain.

Carla Grandori

The Rockefeller University,

Box 102, 1230 York Ave,

New York,

New York 10021, USA

1. Hardie, G. Nature $\mathbf{3 3 5}, 592-593$ (1988).

2. Brugge, J.S. \& Darrow, D. J. biol. Chem 259, 1550-1557 (1981).

3. Pawson, T. Oncogene 3, 491-495 (1988).

4. Yonemoto, W., Jarvis-Morar, M., Brugge, J.S., Bolen, J.B. \& Israel, M. Proc. natn. Acad. Sci. U.S.A. 82, 4568- 4572 (1985).

5. Bolen, J.B., Rosen, N. \& Israel, M. Proc. natn. Acad. Sci. U.S.A. 82, 7275-7279 (1985)

6. Kato, J.Y. et al. Molec. cell. Biol. 6, 4155-4160 (1986)

7. Potts, W.M., Reynolds, A.B., Lansing, T.J. \& Parsons, T.J. Oncogene Res. 3, 343-355 (1989).

\section{Tale of two serines}

SIR-In a recent paper', Brenner used the fact that serine is encoded by two nonlinked codon types, UCN and AGY, in conjunction with his observation that within several enzyme families catalytic serine residues have different codon representations, to propose that these serines evolved convergently by single substitutions in cysteine or threonine codons (UGY and $\mathrm{ACN}$, respectively), the latter being catalytic residues in ancestral enzymes of each class. This proposal, however, fails to account for the serine codon representations of two groups of proteins.

First, the chymotrypsin-like proteases from Streptomyces griseus, SGPA and SGPB (ref. 2): in these enzymes, the catalytic serine residues are encoded by AGU and UCC, respectively, but the 61 per cent identity of amino acids between SGPA and SGPB, and their similar sizes, make it highly improbable that they belong to two different phylogenetic lineages, as predicted by Brenner's hypothesis. Second, the viral replicative proteins containing the widespread nucleoside-5'-triphosphate (NTP)-binding motif (refs 3-5) Gly-X-X-X-X-Gly-LysSer/Thr (GXXXXGK S/T) where X is any amino acid: as shown in the figure and table, the lineages of the GKS-containing proteins of picornavirus, comovirus and nepovirus derived from serine codon types are incompatible with the phylogeny arising from sequence comparisons (compare $a$ and $b$ in the figure). Moreover, the conspicuous absence in this family of a GKT-containing protein is at odds with Brenner's additional suggestion that, in the case of the NTP-binding motif, threonine might be an evolutionary intermediate between the two kinds of serine, rather than their predecessor. It seems to be the case, in the proteins of this family, that threonine is not acceptable in the NTP-binding motif and that evolutionary transitions between $\mathrm{UCN}$ and AGY 